Nature Reviews Genetics | AOP, published online 26 February 2013; doi:10.1038/nrg3450

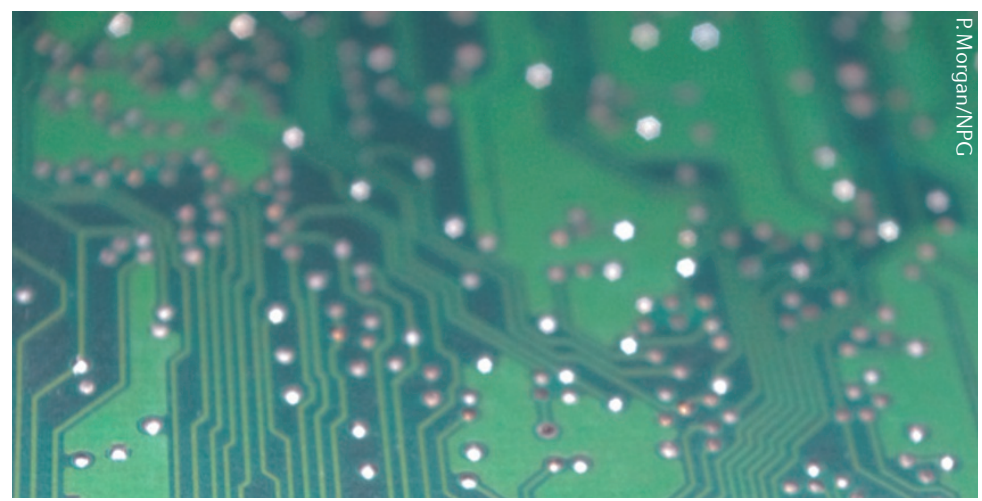

$\Rightarrow$ SYNTHETIC BIOLOGY

\title{
A circuit to remember
}

One challenge of synthetic biology is to assemble genetic circuits efficiently that can carry out complex logic processing functions. Another is to combine processing and memory in a single device. A recent study addresses both challenges by making use of recombinase enzymes to build integrated logic and memory devices in bacterial cells.

Synthetic devices that carry out complex logic processing can be made by putting together several different logic gates, but this is rather an inefficient process. Siuti and colleagues devised a simpler alternative method using two bacterial recombinases that have distinct recognition sites and that were put under the control of promoters that respond to different input signals. Depending on the orientation of pairs of recognition sites, the recombinases catalyse either inversion or excision of DNA in an irreversible manner. The authors made constructs in which various promoters, terminators and output genes can be inverted by the recombinases. By putting these together in various combinations, they made circuits that encoded all 16 Boolean logic functions (ranging from simple AND gates to the most complex functions). This construction requires fewer steps and components than do existing strategies.

Importantly, these circuits also incorporate stable memory. Because inversion by the recombinases is irreversible, a stable output is maintained even when the inputs are no longer present. The authors demonstrated this by showing that an AND gate gave a stable ON output for more than 90 generations (9 days) after the inputs were withdrawn. Furthermore, the computational states of these circuits can be determined by sequencing to see whether the recombinases have catalysed inversion, even after the cells have died.

So what can these integrated memory and logic circuits be used for? The authors suggest that one application might be to make biosensors for which high-throughput sequencing can be used to determine the computational state of the biosensors and therefore the conditions they were exposed to. They also demonstrate that these circuits can be used to convert binary inputs to adjustable gene expression outputs. This function could prove to be useful in biotechnology for biomolecule production: the cost of compounds used to induce production could be minimized because the integrated logic and memory devices would require only transient exposure to the inducers to achieve stable expression.

Goals for the future include increasing the complexity of the recombinase-based circuits for a greater range of applications and implementing these circuits for use in eukaryotes.

Louisa Flintoft

ORIGINAL RESEARCH PAPER Siuti, P., Yazbek, J. \& Lu, T. K. Synthetic circuits integrating logic and memory in living cells. Nature Biotech. 10 Feb 2013 (doi:10.1038/nbt.2510)

FURTHER READING Slusarczyk, A. L., Lin, A. \& Weiss, R. Foundations for the design and implementation of synthetic genetic circuits. Nature Rev. Genet. 13, 406-420 (2012) 